Journal of the Operations Research

Society of Japan

Vol. 33, No. 4, December 1990

\title{
THE OUTPUT RATE OF A PARALLEL QUEUEING MODEL WITH OVERLAPPING PROCESSING TIME
}

\author{
Li-Wen Liu Masashi Kowada Koichi Adachi \\ Nagoya Institute of Technology
}

(Received July 25, 1989; Revised April 23, 1990)

\begin{abstract}
The purpose of this paper is to study the output rate of a main-line in a production system which is related with a sub-line. The system consists of one operator and two lines such that the units of type 1 are processed on the main-line and the units of type 2 are processed on the sub-line. Processing time of each unit has two components: handwork and machining. The operator is needed only during the handwork portion of the processing, and can walk from one line to the other instantaneously. Two queueing disciplines are investigated: alternate discipline and preemptive priority discipline. In each case the relationship between the mean output rate of the main-line and the behavior of the sub-line is obtained under the following assumptions: the handwork times and machining times are mutually independent, exponentially distributed random variables; there is an unlimited supply of the units of type 1 in the main-line; and, the units of type 2 arrive according to a Poisson process and queue up in front of the sub-line.
\end{abstract}

\section{Introduction}

An important class of production systems, which arises in manufacturing environment, is one where a single operator alternately attends to two or more parallel lines. In such cases, each unit to be processed in production systems may depart after receiving two kinds of processing: handwork by an operator and automatic machining. Along with the remarkable progress of engineering techniques and the development of automatic level, studying the performance of such systems has increased its importance. We, however, note that although there have been a lot of reports on production systems problems using queueing theory, including the patrolling problems, transfer line problems and flexible manufacturing systems, almost nothing on parallel lines problems with two kinds of processing mentioned as above has been done as queueing models.

To clarify this situation, this paper discusses a such production system as a queueing model. In this system, one operator attends to two lines: a main-line and sub-line. Units to be processed on the main-line (hereafter referred to as units of type 1) are in great demand, and there is an unlimited supply of those in the main-line. While units to be processed on the sub-line (hereafter refereed to as units of type 2) are in temporary demand, and there is a random supply of those in the sub-line. Processing time of each unit, both of type 1 and type 2, has two components: handwork and automatic machining. Upon departure of a unit from a line, a new unit is allowed to enter. The operator is needed only during the handwork portion of each unit's processing, and can walk from one line to the other instantaneously.

For such a production system, although the several performance measures can be considered to answer questions pertaining to the efficiency of the system, our purpose in this paper is mainly to study the output of the main-line and show the relationship between the mean output rate of the main-line and the behavior of the sub-line for the different queueing disciplines. 
We assume that the handwork times and machining times of the units of type $i$ are exponentially distributed with parameters $\mu_{i}$ and $\gamma_{i}$ respectively $(i=1,2)$, and the units of type 2 arrive according to a Poisson process with a parameter $\lambda$ and queue up in front of the sub-line. These processing times are mutually independent, and are independent of the arrival times of the units of type 2. Two queueing disciplines are investigated:

(1) alternate discipline, in which completing the handwork of a unit in a line, the operator can attend to either of the lines next which becomes waiting handwork state earlier; and

(2) preemptive priority discipline, in which the units of type 2 are given high priority, the operator interrupts the handwork of a unit of type 1 and goes to the sub-line as long as there is a requirement from the units of type 2 .

We determine the mean output rate of the main-line in two cases respectively.

It should be pointed that a preemptive priority discipline in which the units of type 1 are given high priority is also practicable. In this case the mean output rate of the main-line is, obviously, equal to its service rate $\mu_{1} \gamma_{1} /\left(\mu_{1}+\gamma_{1}\right)$ since the number of the units of type 1 waiting for being processed is unlimited. So this case does not require special treatment. Furthermore, it may be reasonable to consider the alternate priority of $k$-limited type in which the operator continues to serve without interruption in one line until $k(0<k<\infty)$ units are treated or until the line become empty, whichever happens first. We shall take it as a open question for future research.

The important feature of this model is that although there is only one operator in the system, the two lines may be occupied at the same time. This happens when either machining is going on in both the lines, or when one unit is being machined and another being handworked. Such simultaneous occupation increases the difficulties of mathematical analysis, and few models with such double occupation have been reported. On the while, the problem discussed in this paper can be considered as an adaptation of general twoqueue, single-server models without double occupation which have been studied by several investigators (see, for example, Eisenberg [1], Takacs [6], Sykes [5] and Takagi [7]).

Mean output rate $\sigma$ is an important measure of the efficiency of a production line. This rate is defined by

$$
\sigma=\lim _{t \rightarrow \infty} E[N(t)] / t
$$

where $N(t)$ is the number of units released from a line in the time interval $(0, t]$. For our system, an equivalent definition of the mean output rate of the main-line is, given a steadystate

$$
\sigma=1 / E[U]
$$

where $U$ is the interdeparture time in the main-line (see Muth [2]). This enables us to express the mean output rate of the main-line as

$$
\sigma=\mu_{1}\left(1 / \mu_{1}\right) E[U]
$$

Here $\left(1 / \mu_{1}\right) / E[U]$ is the long-run fraction of time during which the main-line is in the handwork state; namely, the probability that the main-line is in the handwork state when the system is in the steady-state. Hence, at first, we must derive such state probabilities to obtain the mean output rates of the main-line. Section 2 is devoted to such preliminary results. The main results of this paper are given in section 3 . 


\section{Preliminaries}

In this section we derive the required state probabilities and the steady-state existence condition of the system by analyzing a Markov process describing the state of the systcm at time $t$. The number of the units of type 1 present in the system can be considered as infinite since there is a unlimited supply of the units of type 1 in the main-line. So we characterize the state of the system by the number of the units of type 2 and the states of the two lines.

Let $Q(t)$ be the number of the units of type 2 present in the system at time $t, M_{1}(t)$ be the state of the main-line at time $t$, and $M_{2}(t)$ be the state of the sub-line at time $t$. Every line is at any time in one of three possible states. A line is in the machining state when a unit is receiving machining; it is in the handwork state when a unit is receiving handwork; and it is in the idle state otherwise. In the main-line, the idle state is caused when a unit of type 1 has left the main-line and the handwork on a unit of type 2 in the sub-line has not been completed; and in the sub-line, the idle state is caused either in the similar case as above or when no unit of type 2 is present in the system. Then, since the interarrival times of the units of type 2 and the processing time of two types of units are exponentially distributed, $\left\{Q(t), M_{1}(t), M_{2}(t): t \geq 0\right\}$ is a Markov process. We denote

$$
P_{k}\left(m_{1}, m_{2}\right)=\lim _{t \rightarrow \infty} P\left\{Q(t)=k, M_{1}(t)=m_{1}, M_{2}(t)=m_{2}\right\}
$$

and

$$
m_{1}\left(m_{2}\right)= \begin{cases}d & \text { if the main-line (the sub-line) is in the machining state } \\ s & \text { if the main-line (the sub-line) is in the handwork state } \\ w & \text { if the main-line (the sub-line) is in the idle state. }\end{cases}
$$

We analyze two cases respectively below.

\subsection{Alternate discipline}

In the alternate discipline case, the state-transition-rate diagram associated with the Markov process is as shown in Fig 1. From this we have the following steady-state balance equations of the system:

$$
\begin{aligned}
& -\left(\lambda+\mu_{2}\right) P_{k}(w, s)+\delta(k) \lambda P_{k-1}(w, s)+\gamma_{1} P_{k}(d, s)=0 \\
& -\left(\lambda+\mu_{1}+\gamma_{2}\right) P_{k}(s, d)+\delta(k) \lambda P_{k-1}(s, d)+\mu_{2} P_{k}(w, s)+\gamma_{1} P_{k}(d, d)=0 \\
& -\left(\lambda+\gamma_{1}+\gamma_{2}\right) P_{k}(d, d)+\delta(k) \lambda P_{k-1}(d, d)+\mu_{1} P_{k}(s, d)+\mu_{2} P_{k}(d, s)=0 \\
& -\left(\lambda+\mu_{2}+\gamma_{1}\right) P_{k}(d, s)+\lambda P_{k-1}\left(d, m_{2}(k)\right)+\mu_{1} P_{k}(s, w)+\gamma_{2} P_{k+1}(d, d)=0 \\
& -\left(\lambda+\mu_{1}\right) P_{k}(s, w)+\lambda P_{k-1}(s, w)+\gamma_{2} P_{k+1}(s, d)=0
\end{aligned}
$$

for $k \geq 1$, and

$$
\begin{aligned}
& -\left(\lambda+\gamma_{1}\right) P_{0}(d, w)+\mu_{1} P_{0}(s, w)+\gamma_{2} P_{1}(d, d)=0 \\
& -\left(\lambda+\mu_{1}\right) P_{0}(s, w)+\gamma_{1} P_{0}(d, w)+\gamma_{2} P_{1}(s, d)=0
\end{aligned}
$$

where $\delta(k)=1$ for $k>1$ and $\delta(k)=0$ for $k=1$, and $m_{2}(k)=s$ for $k>1$ and $m_{2}(k)=w$ for $k=1$.

Introducing the generating functions

$$
P_{\left(m_{1}, m_{2}\right)}(z)=\sum_{k=0}^{\infty} P_{k}\left(m_{1}, m_{2}\right) z^{k},
$$


the equations (2.3) then can be written in matrix form

$$
A(z) p(z)=b P_{0}(d, w)
$$

where

$$
\begin{aligned}
& \text { where } \\
& p(z)=\left[\begin{array}{c}
P_{(w, s)}(z) \\
P_{(s, d)}(z) \\
P_{(d, d)}(z) \\
P_{(d, s)}(z) \\
P_{(s, w)}(z)
\end{array}\right], \\
& A(z)=\left[\begin{array}{ccccc}
\lambda z-\lambda-\mu_{2} & 0 & \gamma_{1} & 0 \\
\mu_{2} & \lambda z-\lambda-\mu_{1}-\gamma_{2} & \gamma_{1} & 0 \\
0 & \mu_{1} & \lambda z-\lambda-\gamma_{1}-\gamma_{2} & \mu_{2} & 0 \\
0 & 0 & \gamma_{2} / z & \lambda z-\lambda-\mu_{2}-\gamma_{1} & \mu_{1} \\
0 & \gamma_{2} / z & 0 & 0 & \lambda z-\lambda-\mu_{1}-s
\end{array}\right]
\end{aligned}
$$

and

$$
b=\left[\begin{array}{c}
\gamma_{1} \\
0 \\
\mu_{2} \\
-\mu_{2} \\
-\gamma_{1}
\end{array}\right]
$$

Hereafter we denote the $i$-th element of $p(z)$ by $P_{i}(z)$ for convenience.

Let $|A|$ be the determinant of a matrix $A$, and let $A_{i}(z)$ be a matrix obtained by replacing the $i$-th column of $A(z)$ with $b$, we have the following result.

Lemma 1:

If $\rho_{1}=\lambda\left[\frac{1}{\gamma_{2}}+\frac{1}{\mu_{2}}+\frac{\gamma_{1}\left(\mu_{2}+\gamma_{1}+\gamma_{2}\right)}{\left(\mu_{1}+\gamma_{1}+\gamma_{2}\right)\left(\mu_{2}+\gamma_{1}\right)} \cdot \frac{1}{\mu_{1}}\right]<1$, then the steady-state distribution $P_{k}\left(m_{1}, m_{2}\right)$ exists and its generating functions are given by

$$
P_{i}(z)=P_{0}(d, w)\left|A_{i}(z)\right| /|A(z)|, \quad i=1,2, \cdots, 5
$$

where

$$
P_{0}(d, w)=\left(1-\rho_{1}\right) \mu_{1} /\left(\mu_{1}+\gamma_{1}\right)
$$

Proof: We first examine values of $z$ at which $A(z)$ is nonsingular. It is easy to show that at $z=1,|A(z)|=0$, and also $\left|A_{i}(z)\right|=0, i=1,2, \cdots, 5$. Thus, we can write

$$
\begin{aligned}
& |A(z)|=(1-z) Q(z), \\
& \left|A_{i}(z)\right|=(1-z) Q_{i}(z)
\end{aligned}
$$

where $Q(z)$ and $Q_{i}(z)$ are some polynomials. We investigate the zeros of $Q(z)$. These are the roots of the equation $z=K(z)$ where

$$
K(z)=\frac{\gamma_{2} \mu_{2}}{\left(\lambda z-\lambda-\mu_{2}\right)\left(\lambda z-\lambda-\gamma_{2}\right)}\left\{1+\frac{\gamma_{1}(\lambda z-\lambda)\left(2(\lambda z-\lambda)-\gamma_{1}-\gamma_{2}-\mu_{2}\right)}{\left(\lambda z-\lambda-\gamma_{2}-\gamma_{2}-\mu_{1}\right)\left(\lambda z-\lambda-\gamma_{1}-\mu_{2}\right)\left(\lambda z-\lambda-\mu_{1}\right)}\right\}
$$


Now let us consider the function $f(z)=K(z) / z$. We have $f(0)=\infty, f(1)=1, f^{\prime}(1)=$ $K^{\prime}(1)-1$ and $f^{\prime \prime}(z)>0$. Thus the equation $z=K(z)$ has a root $\xi$ such that $0<\xi<1$ if and only if $K^{\prime}(1)>1$. so that if $K^{\prime}(1) \leq 1, Q(z) \neq 0$ for all $z$ in the $|z| \leq 1$. We find from (2.11) that

$$
K^{\prime}(1)=\lambda\left[\frac{1}{\gamma_{2}}+\frac{1}{\mu_{2}}+\frac{\gamma_{1}\left(\mu_{2}+\gamma_{1}+\gamma_{2}\right)}{\left(\mu_{1}+\gamma_{1}+\gamma_{2}\right)\left(\mu_{2}+\gamma_{1}\right)} \cdot \frac{1}{\mu_{1}}\right]
$$

Furthermore, if a steady-state distribution exists, then we have $\sum_{i=1}^{5} P_{i}(1)+P_{0}(d, w)=1$. Hence, we get

$$
P_{0}(d, w)=\left(1-K^{\prime}(1)\right) \mu_{1} /\left(\mu_{1}+\gamma_{1}\right) .
$$

This shows that if $K^{\prime}(1)=1$, the $P_{0}(d, w)=0$, and $P_{i}(z)=0$ for $i=1,2, \cdots, 5$. Summarizing these results, the conclusion (2.9) is obtained.

\section{Remark 1:}

We can further consider the significance of $\rho_{1}$. Let $V$ be the total service time of a unit of type 2 , a length of time from the instant at which the unit of type 2 starts to receive handwork to the instant at which the next unit of type 2 starts to receive handwork if any units of type 2 are present. Let $S_{2}$ and $D_{2}$ be the handwork time and the machining time of a unit of type 2 respectively. Obviously, $V=S_{2}+D_{2}$ if the main-line is in the machining state when the machining of a unit of type 2 was completed, and $V=S_{2}+D_{2}+W$ if the operator is treating a unit of type 1 in the main-line when the machining of a unit of type 2 was completed; where $W$ is the remaining handwork time of the unit of type 1 . Thus the expectation of $V$ can be expressed as

$$
E[V]=E\left[S_{2}\right]+E\left[D_{2}\right]+E[W] P\left\{X_{W}=1\right\}
$$

where $P\left\{X_{W}=1\right\}$ is the probability that the operator is treating a unit of type 1 in the main-line when the machining of a unit of type 2 was completed. The random variable $W$ has the same distribution with the handwork times of the units of type 1 since the handwork times of the units of type 1 have a exponential distribution. So we have

$$
E[V]=\frac{1}{\gamma_{2}}+\frac{1}{\mu_{2}}+\frac{1}{\mu_{1}} P\left\{X_{W}=1\right\} .
$$

Furthermore, the probability $P\left\{X_{W}=1\right\}$ is the long-run fraction of time that the main-line is in the handwork state when the sub-line is in the machining state. This implies

$$
\begin{aligned}
P\left\{X_{W}=1\right\} & =P_{2}(1) /\left\{P_{2}(1)+P_{3}(1)\right\} \\
& =\left|A_{2}(1)\right| /\left\{\left|A_{2}(1)\right|+\left|A_{3}(1)\right|\right\} \\
& =\frac{\gamma_{1}\left(\mu_{2}+\gamma_{1}+\gamma_{2}\right)}{\left(\mu_{1}+\gamma_{1}+\gamma_{2}\right)\left(\mu_{2}+\gamma_{1}\right)}
\end{aligned}
$$

It follows that $\rho_{1}=\lambda E[V]$.

\subsection{Preemptive priority discipline}

The preemptive priority case is easier to analyze than the alternate discipline case. One reason is that the queue formed in the sub-line can be considered as a standard $M / G / 1$ queue with a Poisson input of a parameter $\lambda$ and with mutually independent service times $V$; where $V$ is the sum of the handwork time and the machining time of a unit of type 2 . It immediately follows that if $\rho_{2}=\lambda\left(\frac{1}{\gamma_{2}}+\frac{1}{\mu_{2}}\right)<1$, then the Markov process $\left\{Q(t), M_{1}(t), M_{2}(t): t \geq 0\right\}$ has 
a unique steady-state distribution. Another reason is that the state of the system for this case, as shown in Fig 2, is simpler than that of the system for the alternate discipline case. In the same way as section 2.1, the generating functions of the steady-state distribution for this case can be written as

$$
A(z) p(z)=b(z)
$$

where

$$
\begin{gathered}
p(z)=\left[\begin{array}{c}
P_{(w, s)}(z) \\
P_{(s, d)}(z) \\
P_{(d, d)}(z) \\
P_{(d, s)}(z)
\end{array}\right], \\
A(z)=\left[\begin{array}{cccc}
\lambda z-\lambda-\mu_{2} & \gamma_{2} / z & 0 & \gamma_{1} \\
\mu_{2} & \lambda z-\lambda-\mu_{1}-\gamma_{2} & \gamma_{1} & 0 \\
0 & \mu_{1} & \lambda z-\lambda-\gamma_{1}-\gamma_{2} & \mu_{2} \\
0 & 0 & \gamma_{2} / z & \lambda z-\lambda-\mu_{2}-\gamma_{1}
\end{array}\right]
\end{gathered}
$$

and

$$
b(z)=\left[\begin{array}{c}
\left(\lambda+\mu_{1}-\lambda z\right) P_{0}(s, w)-\gamma_{1} P_{0}(d, w) \\
0 \\
0 \\
\left(\lambda+\gamma_{1}-\lambda z\right) P_{0}(d, w)-\mu_{1} P_{0}(s, w)
\end{array}\right] .
$$

It is clear that if $\rho_{2}<1$, then

$$
\begin{aligned}
& P_{0}(s, w)+P_{0}(d, w)=1-\rho_{2}, \\
& P_{0}(s, w)=\left(1-\rho_{2}\right) \gamma_{1} /\left(\mu_{1}+\gamma_{1}\right) \\
& P_{0}(d, w)=\left(1-\rho_{2}\right) \mu_{1} /\left(\mu_{1}+\gamma_{1}\right)
\end{aligned}
$$

For the steady-state distribution of the process, we have the following result. The notations used here are the same with those in section 2.1 .

\section{Lemma 2:}

If $\rho_{2}<1$, then the steady-state distribution $P_{k}\left(m_{1}, m_{2}\right)$ exists and its generating functions are given by

$$
P_{i}(z)=\left|A_{i}(z)\right| /|A(z)|, \quad i=1,2,3,4
$$

Proof: All we have to do is to examine whether $A(z)$ is nonsingular. Noting that at $z=1,|A(z)|=0$, and $\left|A_{i}(z)\right|=0$ for $i=1,2,3,4$, we can write

$$
\begin{aligned}
& |A(z)|=(1-z) Q(z), \\
& \left|A_{i}(z)\right|=(1-z) Q_{i}(z), \quad i=1,2,3,4 .
\end{aligned}
$$

The polynomial $Q(z)$ is expressed by $Q(z)=f(z) g(z)$ where

$$
\begin{aligned}
& f(z)=\lambda\left(\lambda+\gamma_{2}-\lambda z\right)+\mu_{2}\left(\lambda-\gamma_{2} / z\right) \\
& g(z)=\left(\lambda+\mu_{2}+\gamma_{1}-\lambda z\right)\left(\lambda+\mu_{1}+\gamma_{1}+\gamma_{2}-\lambda z\right)-\mu_{2} \gamma_{2} / z
\end{aligned}
$$


The function $f(z)$ has two zeros, namely,

$$
\begin{aligned}
& z_{1}=\frac{\lambda+\mu_{2}+\gamma_{1}+\sqrt{\left(\lambda+\mu_{2}+\gamma_{2}\right)^{2}-4 \mu_{2} \gamma_{2}}}{2 \lambda}>1 \\
& z_{2}=\frac{\lambda+\mu_{2}+\gamma_{1}-\sqrt{\left(\lambda+\mu_{2}+\gamma_{2}\right)^{2}-4 \mu_{2} \gamma_{2}}}{2 \lambda}>1 \quad \text { if } \rho_{2}<1 .
\end{aligned}
$$

While, for $|z|=1$, we have

$$
\left|\left(\lambda+\mu_{2}+\gamma_{1}-\lambda z\right)\left(\lambda+\mu_{1}+\gamma_{1}+\gamma_{2}-\lambda z\right)\right|>\left|\mu_{2} \gamma_{2} / z\right|
$$

so that by Rouche's theorem, $g(z)$ has no zero in $|z|<1$.

Consequently, (2.19) is obtained.

\section{Main results}

Using the lemmas obtained in section 2 , we have the following results.

Theorem 1:

In the alternate discipline case, if $\rho_{1}=\lambda\left[\frac{1}{\gamma_{2}}+\frac{1}{\mu_{2}}+\frac{\gamma_{1}\left(\mu_{2}+\gamma_{1}+\gamma_{2}\right)}{\left(\mu_{1}+\gamma_{1}+\gamma_{2}\right)\left(\mu_{2}+\gamma_{1}\right)} \cdot \frac{1}{\mu_{1}}\right]<1$, then the mean output rate $\sigma_{1}$ of the main-line is given by

$$
\sigma_{1}=\frac{\gamma_{1} \mu_{1}}{\gamma_{1}+\mu_{1}}\left(1-\lambda \frac{\gamma_{1}}{\mu_{2}\left(\mu_{2}+\gamma_{1}\right)}\right)
$$

Theorem 2:

In the preemptive priority case, if $\rho_{2}=\lambda\left[\frac{1}{\gamma_{2}}+\frac{1}{\mu_{2}}\right]<1$, then the mean output rate $\sigma_{2}$ of the main-line is given by

$$
\sigma_{2}=\frac{\gamma_{1} \mu_{1}}{\gamma_{1}+\mu_{1}}\left(1-\lambda \frac{\gamma_{1}\left(\mu_{1}+\gamma_{1}+\mu_{2}+\gamma_{2}\right)}{\mu_{2}\left[\mu_{1} \mu_{2}+\gamma_{1}\left(\mu_{1}+\gamma_{1}+\mu_{2}+\gamma_{2}\right)\right]}\right) .
$$

Proof of theorem 1:

Since $\left(1 / \mu_{1}\right) / E[U]$ is the probability that the main-line is in the handwork state, we have

$$
\left(1 / \mu_{1}\right) / E[U]=P_{2}(1)+P_{5}(1)
$$

Similarly,

$$
\left(1 / \gamma_{1}\right) / E[U]=P_{3}(1)+P_{4}(1)+P_{0}(d, w) .
$$

Using $\sum_{i=1}^{5} P_{i}(1)+P_{0}(d, w)=1$, we have

$$
\sigma_{1}\left(1 / \gamma_{1}+1 / \mu_{1}\right)=1-P_{1}(1)
$$

and by calculating $P_{1}(1)$ from (2.9), we finally obtain (3.1).

\section{Proof of theorem 2:}

This is similar to that of Theorem 1, and is omitted. 


\section{Remark 2:}

1. In the alternate discipline case, Theorem 1 shows that the mean output rate $\sigma_{1}$ of the main-line has a linear relationship with the mean input rate $\lambda$ of the sub-line for the steady-state system. The reason that $\sigma_{1}$ decreases with the increasing of $\lambda$ is because the idle time of the main-line waiting for the operator becomes longer with the increasing of $\lambda$. As $\lambda \rightarrow 0$, the system reduces to a one-queue, single server system with sufficient the units of type 1 , and $\sigma_{1}$ reaches it's maximum. It is also natural that $\sigma_{1}$ has no relation to $\gamma_{2}$ in this case, since the idle state of the main-line is caused only by the handworks of the units of type 2.

2. In the preemptive priority case, Theorem 2 shows that the mean output rate $\sigma_{2}$ of the main-line also has a linear relationship with $\lambda$. However, contrary to the alternate discipline case, $\sigma_{2}$ has relation to $\gamma_{2}$ in this case. This is because $\sigma_{2}$ is dependent on the total service times of the units of type 2 .

3. We can compare $\sigma_{2}$ and $\sigma_{1}$ by rewriting $\sigma_{2}$ as

$$
\sigma_{2}=\frac{\gamma_{1} \mu_{1}}{\gamma_{1}+\mu_{1}}\left(1-\lambda \frac{\gamma_{1}}{\mu_{2}\left(\mu_{2} \epsilon+\gamma_{1}\right)}\right)
$$

where $\epsilon=\mu_{1} /\left(\mu_{1}+\gamma_{1}+\mu_{2}+\gamma_{2}\right)$. We see that $\sigma_{2}$ is smaller than $\sigma_{1}$. This is because the times of the units of type 1 waiting for handwork are longer in the preemptive priority case than in the alternate discipline case.

Finally, we make mention of the mean output rate of the sub-line. Since the number of the units of type 2 released from the sub-line in the time interval $(0, t]$ is equal to the difference between the number of the units of type 2 arrived in the same time interval and the number of the units of type 2 present in the system at time $t$, the mean output rate of the sub-line is equal to the mean input rate $\lambda$ if the mean number of the units of type 2 present in the system at time $t$ is limited. In this case, the mean output rate of the sub-line is independent of that of the main-line as well as the queueing discipline.

\section{Conclusions and future directions}

This paper has reported the output properties of a queueing model like a production system with overlapping processing time. We have shown that there is a linear relationship between the mean output rate of the main-line and the mean input rate of the sub-line in both the alternate discipline case and in the preemptive priority case. For the relationship between the mean output rate of the main-line and the service of the sub-line, we have shown that the mean output rate of the main-line is only related with the handwork times of the units of type 2 in the alternate discipline case, while the mean output of the main-line is related with both handwork and machining times of the units of type 2 in the preemptive priority case. The output rate of the main-line for the preemptive priority case is smaller than that for the alternate discipline case.

Except for considering such queueing disciplines as $k$-limited type, an extension of this research is on the generalization from the exponential processing times. This does not seem to be easy to analyse. We, however, can consider Erlang type as a first step towards this direction and still the same approach remains to be valid. The model with general handwork times and exponential machining times can be approached by using the imbedded Markov chain. These are open questions for future research. 


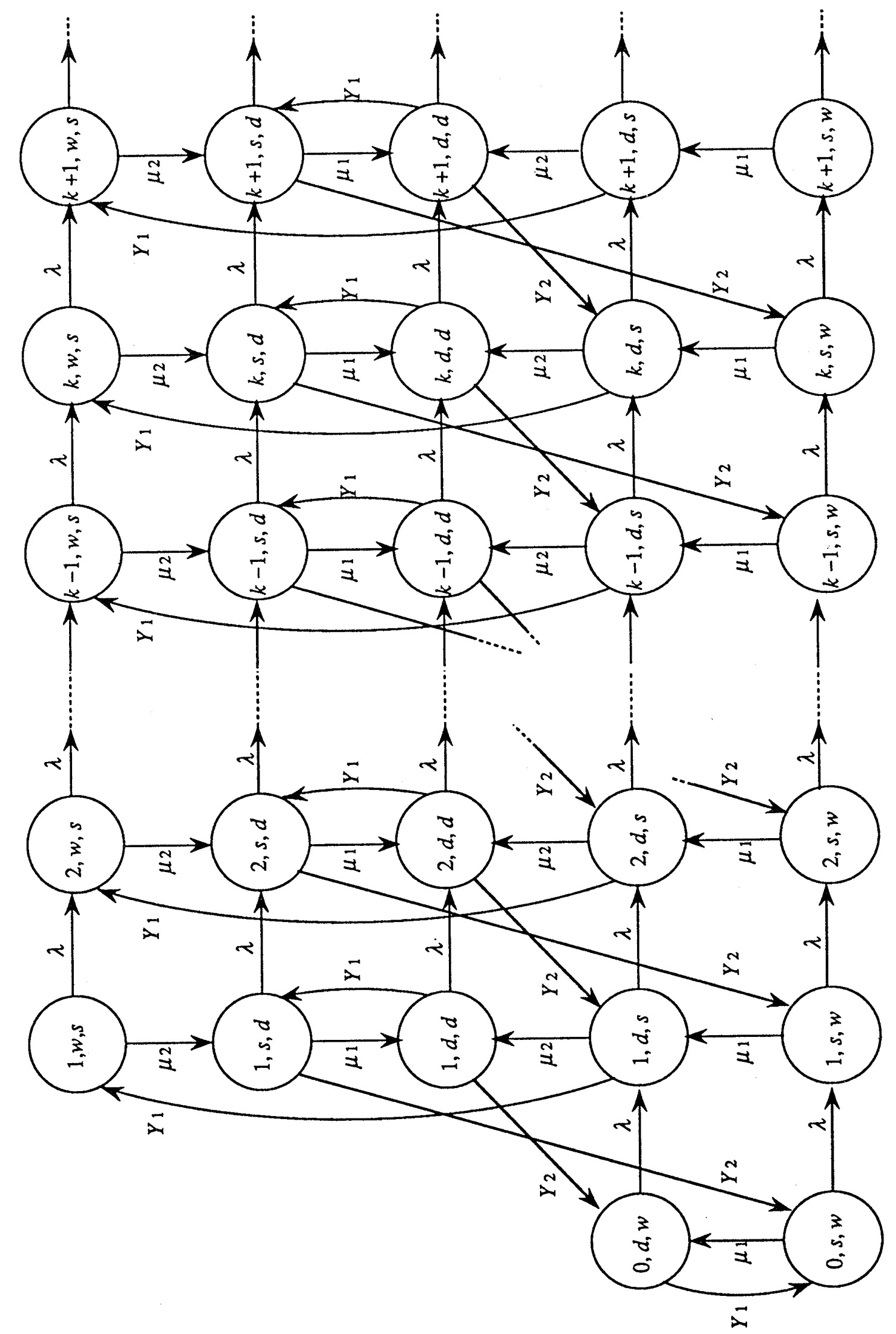

Fig. 1 State-transition-rate diagram for the alternate discipline case. 


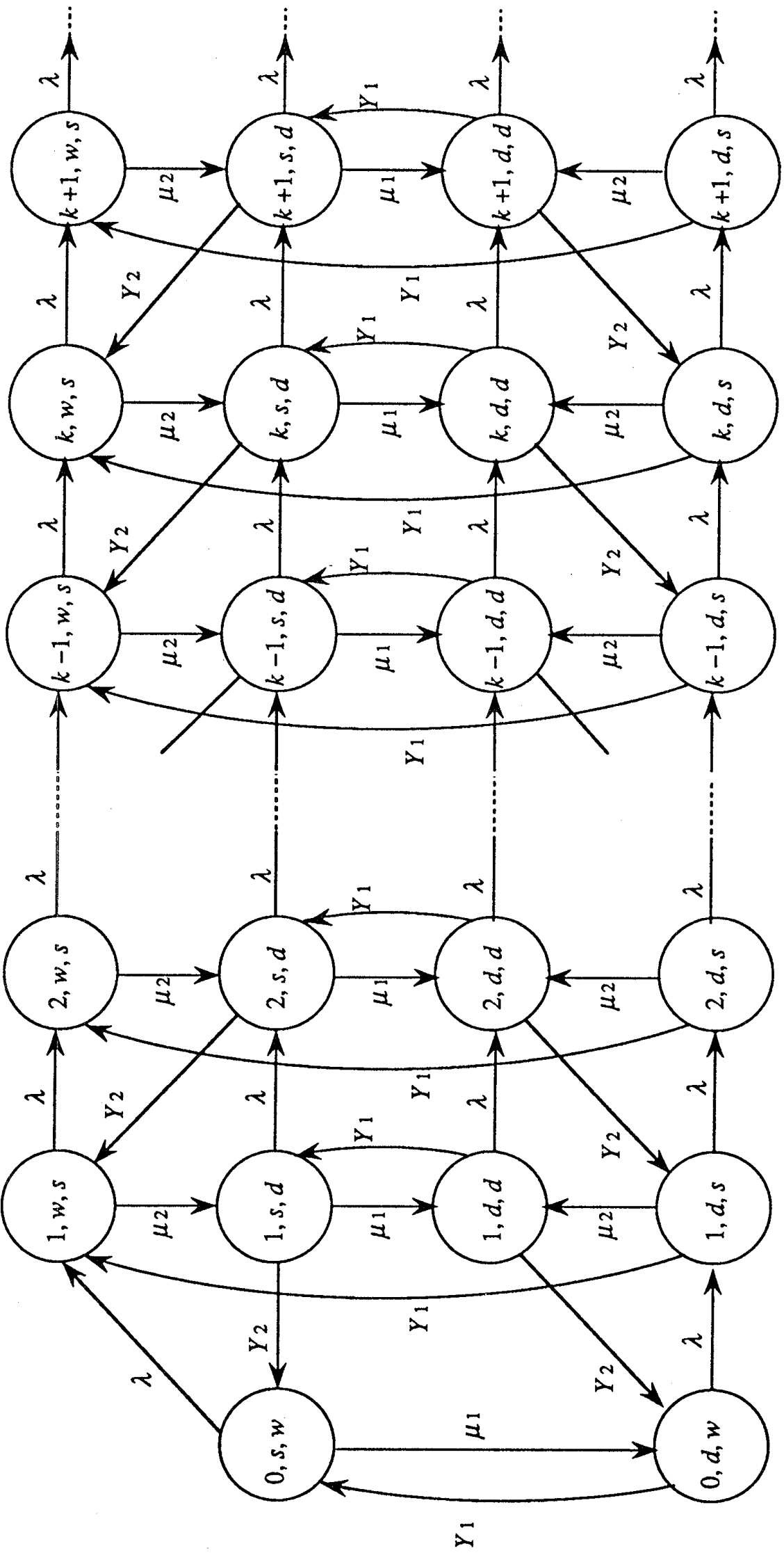

Fig. 2 State-transition-rate diagram for the preemptive priority case. 


\section{References}

[1] Eisenberg, M.: Two Queues with Changeover Times, Opns. Res., Vol. 19, (1971), 386401.

(2) Kowada, M.: Stochastic Process and Their Applications (in Japanese). (1983) Jikkyo.

[3] Muth, E. J.: Stochastic Processes and their Network Representations Associated with a Production Line Queueing Model, Europ. J. of Opnl. Res., Vol. 15, (1984), 63-83.

[4] Prabhu, N. U.: Queues and Inventories: A Study of their Basic Stochastics Process. (1965) John Wiley, New York.

[5] Sykes, J. S.: Simplified Analysis of an Alternating-Priority Queueing Model with Setup Times, Opns. Res., Vol. 18, (1970), 1182-1192.

[6] Takacs, L.: Two Queues Attended by a Single Server, Opns. Res., Vol. 16, (1968), 639-650.

[7] Takagi, H.: Analysis of Polling System. (1986) MIT Press, Cambridge.

Masashi Kowada

Dept. of Systems Engineering

Nagoya Institute of Technology

Nagoya, 466

Japan 